Fluctuation and Noise Letters

(C) World Scientific Publishing Company

\title{
A New Approach to the Treatment of Separatrix Chaos
}

\author{
STANISLAV M. SOSKIN \\ Institute of Semiconductor Physics, 03028 Kiev, Ukraine \\ stanislav.soskin@gmail.com \\ RICCARDO MANNELLA \\ Dipartimento di Fisica, Università di Pisa, 56127 Pisa, Italy \\ mannella@df.unipi.it \\ OLEG M. YEVTUSHENKO \\ Physics Department, Ludwig-Maximilians-Universität München, D-80333 München, Germany \\ bom@ictp.it \\ IGOR A. KHOVANOV \\ School of Engineering, University of Warwick, Coventry CV4 7AL, UK \\ i.khovanov@warwick.ac.uk \\ PETER V. E. McCLINTOCK \\ Physics Department, Lancaster University, Lancaster LA1 4 YB, UK \\ p.v.e.mcclintock@lancaster.ac.uk \\ Received (received date) \\ Revised (revised date)
}

\begin{abstract}
We review an approach to separatrix chaos that has allowed us to solve some significant problems by: (i) finding analytically the maximum width of the chaotic layer, a problem that lay unsolved for 40 years, and showing that the maximum may be much larger than had previously been assumed; (ii) describing the drastic facilitation of the onset of global chaos between neighbouring separatrices, a phenomenon discovered 8 years ago.
\end{abstract}

\section{Introduction}

In this section, we first introduce the concept of separatrix chaos and demonstrate that it constitutes the seed of any chaos in time-periodically perturbed 1D Hamiltonian systems. We then describe some of the problems that stimulated the development of our new approach to separatrix chaos and, finally, explain why it is difficult to use earlier approaches to solve these and related problems.

\subsection{Separatrix chaos}

Hamiltonian dynamics is described by equations of the type: 

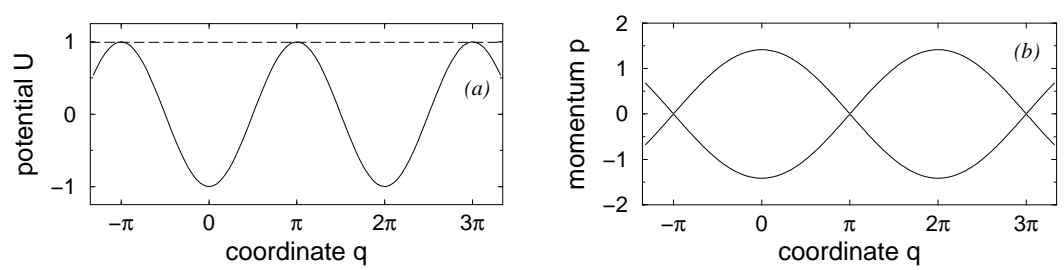

Fig. 1. Pendulum: (a) potential, (b) separatrix in the phase plane.

$$
\begin{aligned}
& \frac{d q_{i}}{d t}=\frac{\partial H}{\partial p_{i}}, \quad \frac{d p_{i}}{d t}=-\frac{\partial H}{\partial q_{i}}, \\
& i=1,2,3, \ldots . \quad H \equiv H\left(q_{1}, q_{2}, \ldots, p_{1}, p_{2}, \ldots, t\right) .
\end{aligned}
$$

The simplest case is a one-dimensional (1D) Hamiltonian $H=H(q, p)$, where $H$ is an integral of motion (the energy $E$ ) and any finite motion is periodic in time:

$$
q(t+T)=q(t), \quad p(t+T)=p(t), \quad T \equiv T(E), \quad \omega \equiv \frac{2 \pi}{T} \equiv \omega(E) .
$$

An important notion is that of a separatrix. Suppose the phase space of the system possesses a saddle, i.e. an unstable stationary point of the hyperbolic type, which two trajectories approach and from which two trajectories depart, exponentially slowly. These four trajectories form the separatrix. As the energy approaches that of the separatrix $E_{s}$, the period of oscillation logarithmically diverges:

$$
\omega^{-1}(E) \propto T(E) \propto \ln \left(1 /\left|E-E_{s}\right|\right), \quad E \approx E_{s} .
$$

The separatrix typically separates trajectories of different topology. An archetypal example is a pendulum (Fig. 1): $H=p^{2} / 2+U(q)$, where $U(q)=-\cos (q)$.

For higher-dimensional Hamiltonians, the motion is typically much more complicated. In this review, we consider the simplest case: $\mathrm{D}=\frac{3}{2}$, i.e. a $1 \mathrm{D}$ Hamiltonian with a time-dependent perturbation. Moreover, we restrict ourselves to the most characteristic case - a weak time-periodic perturbation.

In the 1950s and 60s, Kolmogorov, Arnold and Moser developed a theory (KAMtheory) which says that a majority of perturbed trajectories remain regular: invariant tori are slightly deformed rather than destroyed i.e. the perturbation does not modify the topology of a trajectory in a stroboscopic Poincaré section [1].

However there are two characteristic regions where the KAM theory is inapplicable, so that some of the corresponding trajectories are irregular. The first one relates to the separatrix of the unperturbed system. Due to the divergence of $T(E)$, even a small change of energy caused by the perturbation results in a strong change of the system coordinate after a given interval of time. This causes the separatrix to be replaced by a chaotic layer (Fig. 2), where regions of regular and irregular motion are mixed in a complicated manner. The chaotic layer is a very complicated object: it has been studied in hundreds of papers, starting from [2] in 1967. 


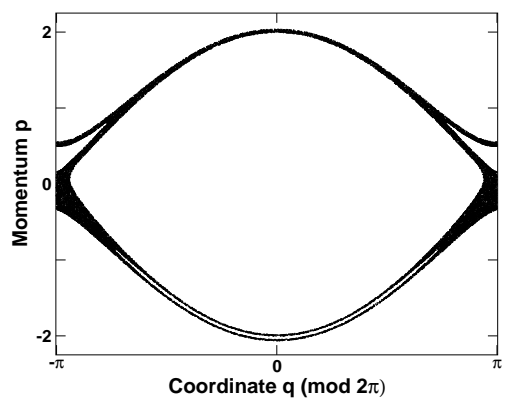

Fig. 2. Stroboscopic Poincare section for the Hamiltonian $H=p^{2} / 2-\cos (q)+0.01 q \cos (t)$.

The second region where the motion may be irregular relates to resonances i.e. to regions where

$$
E \approx E^{(n)}, \quad n \omega\left(E^{(n)}\right)=\omega_{f}, \quad n=1,2,3, \ldots
$$

The slow dynamics of the deviation of action from that at the resonance energy, and of the related slow angle, are governed by the auxiliary (or nonlinear resonance) Hamiltonian, which is typically taken as being pendulum-like [3]. The phase plane of this auxiliary Hamiltonian necessarily possesses a separatrix, but the fast oscillations (neglected in a leading-order analysis of the slow dynamics) destroy it, replacing it with a narrow chaotic layer.

Thus, separatrix chaos plays a key role in the onset of any chaos in a periodically perturbed Hamiltonian system.

\subsection{Stimulating problems}

The development of our approach was stimulated in particular by the following open questions concerning separatrix chaos:

1. How can one describe theoretically the maximal (over the perturbation frequency) width of the separatrix chaotic layer? Neither physicists [4] nor mathematicians [5] had cracked this problem during the 40 years following the pioneering work by Zaslavsky and Filonenko [6].

2. How can one describe theoretically the involvement of resonant motion in separatrix chaos? This problem became topical with the work of Shevchenko in 1998 [7] although it had been implicit since much earlier [3]. We now describe these problems in more detail. The chaotic layer's energy width depends on the ratio $\omega_{f}$ between the perturbation frequency and frequency of small oscillations of the unperturbed system. The three characteristic ranges of $\omega_{f}$ are -

(i) $\omega_{f} \gg 1$ : the width is exponentially small $[4,5]$.

(ii) $\omega_{f} \rightarrow 0$ : in the adiabatic limit, the width is typically ${ }^{\mathrm{a}}$ small $[9,10]$, namely 
of the order of the perturbation amplitude $h$.

(iii) $\omega_{f} \sim 1$ : for the intermediate range qualitative estimates $[4,5]$ suggested a width of $\sim h$. The range of moderately small frequencies remained almost unexplored (perhaps because it was assumed to be uninteresting), with one exception [7] where it was considered heuristically.

Thus, most theoretical estimates suggested that the maximum should occur at $\omega_{f} \sim 1$ where it would have a value of order $h$.

However, numerical studies (e.g. [7]) revealed a different picture: typically, many peaks were seen in the range $\omega_{f} \lesssim 1$, with heights substantially exceeding $h$, suggesting that high peaks actually represent the dominant feature of the dependence of the layer width on the perturbation frequency. The authors of the numerical studies intuitively associated them with nonlinear resonances and an approximate heuristic theory was developed [7] to estimate their positions and maxima. But the mechanism of the involvement of nonlinear resonances in separatrix chaos remained unclear, and a consistent theory of the peaks and a general analysis were lacking.

\subsection{Earlier approaches}

Of the many criteria for chaos, two of those most widely used are:

1. The existence of a positive Lyapunov exponent [11], implying exponential growth in some direction in the separation between initially close trajectories, leading in turn to irregular transport.

2. Destruction of invariant tori (KAM-theory) $[4,5]$, leading in particular to the replacement in the Poincaré section of regular trajectories (lines i.e. 1D objects) by layers (2D objects) where regions with regular and irregular dynamics are mixed in a complicated manner.

Usually, however, Lyapunov exponents have to be found numerically, it being hard to calculate them analytically [11]; and KAM-theory is difficult to apply in the relevant ranges of parameters, where resonances play a crucial role $[1,12]$.

\section{Our approach to separatrix chaos}

\subsection{Our initial tool: the separatrix map}

The separatrix map was introduced in [6] and rederived rigorously in [13]. As an illustration, consider as an example the ac-driven double-well potential system:

$$
H=H_{0}-h q \cos \left(\omega_{f} t\right), \quad H_{0}=p^{2} / 2+U(q), \quad U(q)=-q^{2}+q^{4} .
$$

For the sake of convenience, we refer to $H_{0}(q, p) \equiv E$ as the "energy". Differentiating $E$ with respect to time and using the Hamiltonian equations, we obtain $d E / d t=h \cos \left(\omega_{f} t\right) d q / d t \propto d q / d t$. Thus, energy changes relatively fast only when the velocity $d q / d t$ is relatively large. If $E$ is close to the barrier value, then the 
system spends most of its time near the saddle, where the velocity is near zero, i.e. short pulses of significant velocity are separated by long intervals at almost zero velocity. The duration of a long interval is related to the period of the unperturbed system with the energy possessed by the perturbed system during the interval.

Introducing the angle of the perturbation $\varphi \equiv \omega_{f} t$ and the sign of the velocity $\sigma$, one can map $E$ and $\sigma$ at the beginning of a given pulse, and $\varphi$ in its middle, onto their values in the next pulse: $\left(E_{i}, \varphi_{i}, \sigma_{i}\right) \rightarrow\left(E_{i+1}, \varphi_{i+1}, \sigma_{i+1}\right)$. This map is called the separatrix map (SM). For our model, it is

$$
\begin{aligned}
& E_{i+1}=E_{i}+\sigma_{i} h \epsilon \sin \left(\varphi_{i}\right), \\
& \varphi_{i+1}=\varphi_{i}+\frac{\omega_{f} \pi\left(3-\operatorname{sign}\left(E_{i+1}-E_{s}\right)\right)}{2 \omega\left(E_{i+1}\right)}, \\
& \sigma_{i+1}=\sigma_{i} \operatorname{sign}\left(E_{s}-E_{i+1}\right),
\end{aligned}
$$

where the Melnikov integral $\epsilon \equiv \epsilon\left(\omega_{f}\right) \sim 1$ for the relevant range of $\omega_{f}[4,11]$.

There are both heuristic $[4,11]$ and rigorous [5] criteria for the onset of chaos but almost all of them miss the relevant range of moderately small $\omega_{f}$. The one exception [7] appreciated the role of nonlinear resonances, but the resultant calculations led to values of the maxima that were quantitatively incorrect in the asymptotic $h \rightarrow 0$ limit. The nature of the connections between nonlinear resonances and separatrix chaos remained unclear as did also the question of the generality of the results.

Our new approach [14-16] has allowed us to solve consistently both these and some related problems.

\subsection{Basic elements of the approach}

\subsubsection{Decorrelation of angle}

If the system gets onto the separatrix at the $(i+1)$ th step i.e. $E_{i+1}=E_{s}$, then the change in angle at this step, $\varphi_{i+1}-\varphi_{i}$, diverges due to the divergence of $\omega^{-1}\left(E_{i+1}\right)$ (see Eq. (3)). This decorrelation of angle leads, in turn, to a decorrelation of the energy since $E_{i+1}-E_{i} \propto \sin \left(\varphi_{i}\right)$ while $\sin \left(\varphi_{i}\right)$ may take any value between \pm 1 . Similarly, $\sigma$ is also decorrelated. Obviously, if all variables of the map change randomly at some step, such a trajectory cannot be regular. Thus, a decorrelation of all variables at some step of an area-preserving map is a sufficient condition for chaos in the map; in the SM, such a decorrelation is provided by the decorrelation of angle. We hope and surmise that such a condition can be shown to satisfy earlier definitions of chaos, in particular the existence of a positive Lyapunov exponent.

\subsubsection{Involvement of a resonant motion into the separatrix chaos}

It might at first appear that a resonant dynamics is impossible near the separatrix because of the divergence of $d \omega / d E \propto 1 /\left|E-E_{s}\right|$, given that resonant dynamics requires an approximate constancy of $\omega(E)$. Paradoxically, however, it is possible 
due to the slowness of the logarithmic divergence: if $x$ is small, then $\ln (1 / x)$ remains almost constant even at a rather large variation of $x$.

The energy at some step of the chaotic trajectory takes the value $E_{s}: E_{n}=E_{s}$, while $\varphi_{n}$ and $\sigma_{n}$ may be arbitrary. Consider changes of energy at the next step which have one and the same sign: let it be positive for example. Then $E_{n+1}-$ $E_{s} \sim h\left|\sin \left(\varphi_{n}\right)\right|$. The properties of the logarithmic function mean that $\omega\left(E_{n+1}\right) \simeq$ $\omega\left(E_{s}+h\right)$ for almost all values of $\varphi_{n}$ (except in the near multiples of $\pi$ ). Moreover, $\omega(E)$ remains approximately constant for rather large further variation of $E-E_{s}$ of the same sign. Thus, if $\omega_{f} \approx \omega\left(E_{s}+h\right)$, a resonant dynamics should be relevant.

By reducing the discrete map equations to the differential equations of motion for the resonance Hamiltonian, we were able to prove the above intuitive ideas explicitly within the relevant range of energies.

\subsubsection{Resonant dynamics near the separatrix}

The conventional approximation by which resonant dynamics is described by an auxiliary pendulum-like (Chirikov) Hamiltonian $[3,4,7,11]$ is valid only if $|d \omega / d E|$ is approximately constant within the relevant range of energy. This is not the case near the separatrix, where $|d \omega / d E| \propto 1 /\left|E-E_{s}\right|$ varies rapidly. We have developed the first analytic description of the resonant dynamics near the separatrix.

From the quantitative point of view, this part of our approach is of particular importance because resonant dynamics provides the leading term of our asymptotic theory describing the boundaries of the chaotic layers and related quantities in the relevant range of the perturbation frequency.

\section{Application to the single-separatrix layer}

As an illustrative example, we use the paradigmatic case of an ac-driven pendulum $H=p^{2} / 2-\cos (q)+h q \cos \left(\omega_{f} t\right)$, comparing numerical results with earlier estimates and with our theory. Consider the deviation of the lowest energy in the layer $E_{l}$ from the separatrix value $E_{s}$. Fig. 3 presents numerical calculations of the deviation $\Delta E^{(-)} \equiv\left(E_{s}-E_{l}\right)$ normalized by the perturbation amplitude, as a function of frequency, for different values of $h$. One can see a clear growth of the maximum as $h$ decreases. The inset compares these results with earlier estimates, with which they clearly disagree. In contrast, our asymptotic theory [15] fits the numerical data well, as shown in Fig. 4: the paper also classified all systems in the context of the functional dependence of the width on $h$ and considered characteristic examples. Note also that our theory describes well, not only the width of the layer, but also its boundaries in the Poincaré section.

\section{Application to global chaos between separatrices}

If an unperturbed system possesses two or more separatrices, then a sufficiently large periodic perturbation gives rise to dynamic (chaotic) transport connecting 


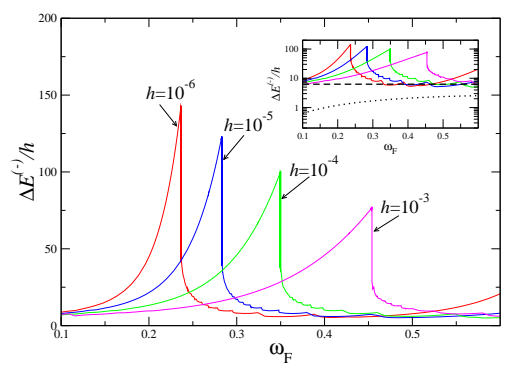

Fig. 3. Deviation of the lowest energy in the layer from the separatrix value, normalized by $h$, as function of frequency, for different $h$. The inset (note the logarithmic ordinate) compares the same results (full curves) with earlier predictions (dashed $[4,10]$ and dotted [17] lines).
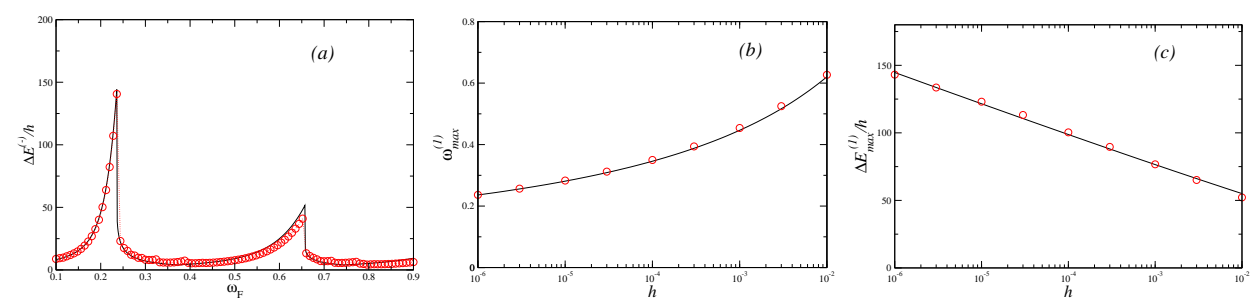

Fig. 4. Comparison of our theory (solid line) and numerical data (circles): (a) $\Delta E^{(-)} / h$ as function of $\omega_{f}$ for $h=10^{-6}$; (b) position of the first maximum as function of $h$; (c) value of the first maximum as function of $h$.

neighbouring separatrices, a phenomenon that is important in many physical applications. Conventionally, the scenario for onset is as follows. For small perturbation amplitude $h$, the separatrices are replaced by thin chaotic layers. As the amplitude grows, the layers widen and, at some critical value $h_{c r}$, they merge, marking the onset of global chaos. Typically, $h_{c r}$ is of the order of the difference of energies at the separatrices. However, it was discovered in 2003 [18] that $h_{c r}$ may be much lower if the perturbation frequency $\omega_{f}$ is close to particular values. We show this using as an example the unperturbed model illustrated in Fig. 5:

$$
H_{0}=p^{2} / 2+U(q), \quad U(q)=(\Phi-\sin (q))^{2} / 2, \quad \Phi=0.2 .
$$

Fig. 6(a) shows a bifurcation diagram of the system with $H=H_{0}+h q \sin \left(\omega_{f} t\right)$ in the plane of the perturbation parameters. The shaded area indicates the parameter range for which global chaos exists. Its lower boundary, representing the function $h_{c r}\left(\omega_{f}\right)$, possesses deep minima (spikes). Their existence can be explained as follows. The dependence of the eigenfrequency on energy $\omega(E)$ (Fig. $5(\mathrm{c})$ ) is very flat (in the asymptotic limit $\Phi \rightarrow 0$, it approaches a rectangular shape). If $\omega_{f}$ is close to the local maximum $\omega_{m}$ of the function $\omega(E)$, then even a weak perturbation remains approximately resonant with eigenoscillations over almost the whole range 

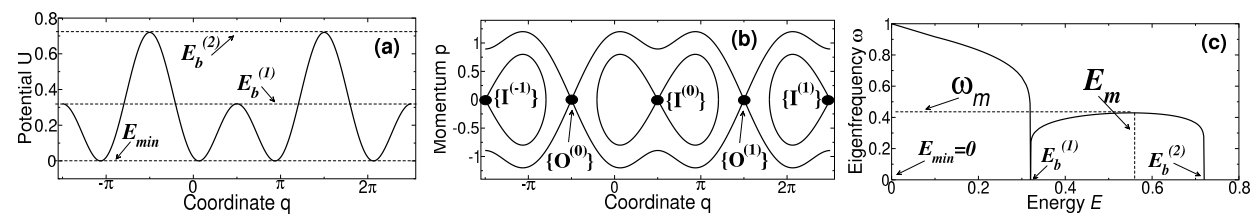

Fig. 5. (a) Potential of Eq. (7). (b) Separatrices in the phase plane. (c) Oscillation frequency as function of energy.
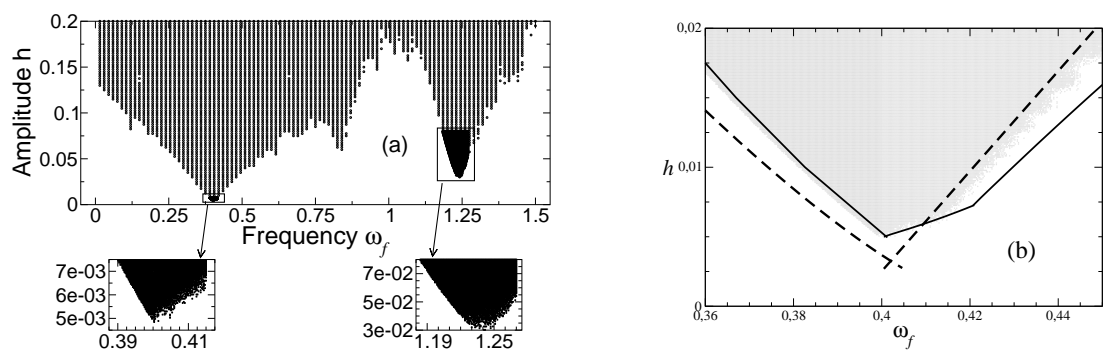

Fig. 6. (a) Bifurcation diagram for $H=H_{0}+h q \sin \left(\omega_{f} t\right)$; (b) the first minimum (shaded) and our theory: dashed line - the leading-order theory, solid line - the theory with higher-order corrections

of energies between the separatrices, so that resonant dynamics may be involved in the separatrix chaos related to each of them, thus providing transport between them. Using our new approach, we developed a consistent asymptotic theory [14] which nicely describes the spikes over a wide vicinity of their minima (Fig. 6(b)).

\section{Some examples of applications and relevant problems}

We can summarise the present status of the new method as follows -

I. The approach itself:

- Evaluates the boundaries of separatrix chaotic layers, including their energy width, for a diversity of perturbed systems with separatrices.

- Promises to be useful for the analysis of transport within the region of the separatrix chaos.

II. Results on the thin single-separatrix layer may:

- Be generalized to describe a wide variety of systems with separatrices.

- Provide for a method [15] to facilitate the overlap of resonances of different orders, in turn facilitating the onset of global chaos.

III. Inter-separatrix transport is likely to be important in, for example:

- Spinning pendula [19] and, in some cases, electron gas in magnetic superlattice [20,21], both being descibed by the model (7).

- Partial stochastic webs, arising if the resonance is approximate rather than exact [4]: there are many separatrices and, the more separatrices are linked by chaotic transport, the larger the web [22] becomes; it is 
relevant to quantum electron transport in semiconductor superlattices in magnetic and electric fields [22,23] when the scattering is very weak.

- Meandering flows [24] e.g. the Gulf Stream [25], where "cross-jet" (inter-separatrix) transport retards transport along the jet.

- Cold atoms in optical lattices [26] can model a variety of potential systems with practically zero dissipation. So, they could model doubleor multi-barrier potentials with barriers of different heights.

- Billiards, which may possess more than one separatrix [27].

- Plasma physics [28].

\section{Conclusions}

Our approach to the treatment of separatrix chaos includes three basic elements: a new criterion for chaos in the separatrix map, i.e. decorrelation of angle; involvement of resonant dynamics into separatrix chaos, explained by the matching of the separatrix map and continuous resonant dynamics; explicit analysis of the resonant dynamics near the separatrix. It has enabled us to solve a long-standing problem related to the maximum width of the separatrix chaotic layer and to describe the drastic facilitation of the onset of global chaos between separatrices for a characteristic example. There are many potential applications of these results.

\section{Acknowledgements}

We gratefully acknowledge support by the Royal Society through an International Joint Project, and by Pisa University within its collaboration agreement with the Institute of Semiconductor Physics (Kiev), and by the DFG through SFB TR-12.

\section{References}

[1] V. I. Arnold, Mathematical Methods of Classical Mechanics (Springer-Verlag, New York, 1978).

[2] N. N. Filonenko, R. Z. Sagdeev and G. M. Zaslavsky, "Destruction of magnetic surfaces by magnetic field irregularities 2", Nucl. Fusion 7 (1967) 253-266.

[3] B. V. Chirikov, "Universal instability of many-dimensional oscillator systems", Phys. Rep. 52 (1979) 263-379.

[4] G. M. Zaslavsky, Physics of Chaos in Hamiltonian Systems (2nd edition) (Imperial Colledge Press, London, 2007).

[5] G. N. Piftankin and D. V. Treshchev, "Separatrix maps in Hamiltonian systems", Russian Math. Surveys 62 (2007) 219-322.

[6] G. M. Zaslavsky and N. N. Filonenko, "Stochastic instability of trapped particles and conditions of application of the quasi-linear approximation", Sov. Phys. JETP 27 (1968) 851-857.

[7] I. I. Shevchenko, "Marginal resonances and intermittent behaviour in the motion in the vicinity of a separatrix", Phys. Scr 57 (1998) 185-191.

[8] S. M. Soskin, O. M. Yevtushenko and R. Mannella, "Divergence of the Chaotic Layer Width in the Adiabatic Limit and Strong Acceleration of the Chaotic Transport in AC Driven Periodic Systems", Phys. Rev. Lett. 95 (2005) 224101. 
[9] A. I. Neishtadt, "Change in adiabatic invariant at a separatrix", Sov. J. Plasma Phys. 12 (1986) 568-573.

[10] Y. Elskens and D. F. Escande, "Slowly pulsating separatrices sweep homoclinic tangles where islands must be small - an extension of classical adiabatic theory", Nonlinearity 4 (1991) 615-667.

[11] A. J. Lichtenberg and M. A. Lieberman, Regular and Chaotic Dynamics (Springer, New York, 1992).

[12] V. Gelfreich, private communication.

[13] V. Rom-Kedar, "Transport rates of a class of two-dimensional maps and flows", Physica D 43 (1990) 229-268.

[14] S. M. Soskin, R. Mannella and O. M. Yevtushenko, "Matching of separatrix map and resonant dynamics, with application to global chaos onset between separatrices", Phys. Rev. E $\mathbf{7 7}$ (2008) 036221.

[15] S. M. Soskin and R. Mannella, "Maximal width of the separatrix chaotic layer", Phys. Rev. E 80 (2009) 066212.

[16] S. M. Soskin, R. Mannella, O. M. Yevtushenko, I. A. Khovanov and P. V. E. McClintock, "A new approach to the treatment of separatrix chaos and its applications", in Hamiltonian Chaos Beyond the KAM Theory (dedicated to George Zaslavsky), eds. A. Luo and V. Afraimovich (HEP-Springer, Berlin, 2010), pp. 51-141 (see also arXiv:0906.3444v1).

[17] G. M. Zaslavsky, R. Z. Sagdeev, D. A. Usikov and A. A. Chernikov, Weak Chaos and Quasi-Regular Patterns (Cambridge University Press, PVEMcC, 1991).

[18] S. M. Soskin, O. M. Yevtushenko and R. Mannella, "Drastic facilitation of the onset of global chaos", Phys. Rev. Lett. 90 (2003) 174101.

[19] A. A. Andronov, A. A. Vitt and S. E. Khaikin, Theory of Oscillators (Pergamon, Oxford, 1966).

[20] O. M. Yevtushenko and K. Richter, "Effect of an ac electric field on chaotic electronic transport in a magnetic superlattice", Phys. Rev. B 57 (1998) 14839-14842.

[21] S. M. Soskin, R. Mannella and P. V. E. McClintock, "Zero-dispersion phenomena in oscillatory systems", Phys. Reports 373 (2003) 247-408.

[22] S. M. Soskin, P. V. E. McClintock, T. M. Fromhold, I. A. Khovanov and R. Mannella, "Stochastic webs and quantum transport in superlattices: an introductory review", Contemporary Phys. 51 (2010) 233-248.

[23] T. M. Fromhold, A. Patanè, S. Bujkiewicz, P. B. Wilkinson, D. Fowler, D. Sherwood, S. P. Stapleton, A. A. Krokhin, L. Eaves, M. Henini, N. S. Sankeshwar and F. W. Sheard, "Chaotic electron diffusion through stochastic webs enhances current flow in superlattices", Nature 428 (2004) 726-730.

[24] D. del-Castillo-Negrete and P. J. Morrison, "Chaotic transport by Rossby waves in shear flow", Phys. Fluids A 5 (1993) 948-965.

[25] R. M. Samelson, "Fluid exchange across a meandering jet", J. Phys. Oceanogr. 22 (1992) 431-440.

[26] R. Gommers, P. Douglas, S. Bergamini, M. Goonasekera, P. H. Jones, F. Renzoni, "Resonant activation in a nonadiabatically driven optical lattice", Phys. Rev. Lett. 94 (2005) 143001.

[27] E. D. Leonel and L. A. Bunimovich, "Suppressing Fermi Acceleration in a Driven Elliptical Billiard", Phys. Rev. Lett. 104 (2010) 224101.

[28] M. C. Firpo and D. Constantinescu, "Study of the interplay between magnetic shear and resonances using Hamiltonian models for the magnetic field lines", Phys. Plasmas 18 (2011) 032506. 